\title{
Classes of exact wave functions for general time-dependent Dirac Hamiltonians in $1+1$ dimensions
}

\author{
A. S. de Castro and A. de Souza Dutra* \\ UNESP-Campus de Guaratinguetá-DFQ, Avenida Dr. Ariberto Pereira da Cunha, 333 Caixa Postal 205, \\ 12516-410 Guaratinguetá, São Paulo, Brazil
}

(Received 28 January 2003; published 20 May 2003)

\begin{abstract}
In this work we construct two classes of exact solutions for the most general time-dependent Dirac Hamiltonian in $1+1$ dimensions. Some problems regarding some formal solutions in the literature are discussed. Finally the possibility of existence of a generalized Lewis-Riesenfeld invariant connected with such solutions is discussed.
\end{abstract}

DOI: 10.1103/PhysRevA.67.054101

PACS number(s): 03.65.Pm, 03.65.Ge

The interest in solving problems involving timedependent systems has attracted the attention of physicists for a long time. This is due to its applicability in the understanding of many problems in quantum optics, quantum chemistry, and other areas of physics [1-11]. In particular, we can cite the case of the electromagnetic field intensities in a Fabry-Pérot cavity [1]. In fact, this kind of problem still represents a line of investigation that attracts the interest of physicists $[10,11]$. However, as has been observed recently by Landim and Guedes [12], the most part of these works deal with nonrelativistic systems. So they tried to bridge this gap by studying the problem of a fermion in the presence of a time-dependent Lorentz vector linear potential in twodimensional space-time. For this they used the so-called Lewis-Riesenfeld invariant operator, in order to guess the wave function that solves the problem. Unfortunately, they just presented a formal solution to the problem under question. In fact, if one considers that in the most part of the works treating the nonrelativistic time-dependent systems, the solution is not formal only for a limited number of time dependences for the potential parameters, it is little bit strange that in Ref. [12] there is no limitation over those parameters. In view of these comments, as a first example, we are going to complete the program initiated by Landim and Guedes, by solving the problem to its end, showing that there exist really some restrictions for a complete solution to this problem. After that, we will show that there exist at least two classes of solutions to the time-dependent Dirac equation in $1+1$ dimensions. This is done considering the most general combination of Lorentz structures for the potential matrix. Then we show that the problem studied in Ref. [12] is only a particular case of one of those classes. Finally we construct the Lewis-Riesenfeld invariant operators, which have some of our solutions as eigenfunctions, and then discuss its possible generalization.

Let us begin with presenting the Dirac equation in $1+1$ dimensions, in the presence of a time-dependent potential for a fermion of rest mass $m$,

$$
H \psi(q, t)=i \frac{\partial \psi(q, t)}{\partial t},
$$

*Email address: dutra@feg.unesp.br

$$
H=\alpha p+\beta m+V_{V}(q, t)+\beta V_{S}(q, t)+\alpha \beta V_{P}(q, t),
$$

where we used $c=\hbar=1$ and $p$ is the momentum operator. $\alpha$ and $\beta$ are Hermitian square matrices satisfying the relations $\alpha^{2}=\beta^{2}=1,\{\alpha, \beta\}=0$. Here we choose $\alpha=\sigma_{1}$ and $\beta$ $=\sigma_{3}$, where $\sigma_{1}$ and $\sigma_{3}$ are the $2 \times 2$ standard Pauli matrices. In this way $\psi$ is a two-component spinor. Furthermore, the subscripts for the terms of potential denote their properties under a Lorentz transformation: $V$ for the time component of the two-vector potential, $S$ and $P$ for the scalar and pseudoscalar terms, respectively. The absence of the space component of the two-vector potential is due to the possibility of its elimination through a gaugelike transformation.

In the particular case considered by Landim and Guedes [12], one has

$$
H=\alpha p+\beta m+f(t) q .
$$

As suggested by them, we will use as an ansatz for the solution the following spinor:

$$
\psi=\left(\begin{array}{c}
M_{1}(t) \\
M_{2}(t)
\end{array}\right) e^{i \eta(t) q}
$$

so that the corresponding coupled equations are

$$
\begin{aligned}
& i \dot{M}_{1}=[(\dot{\eta}+f) q+m] M_{1}+\eta M_{2}, \\
& i \dot{M}_{2}=[(\dot{\eta}+f) q-m] M_{2}+\eta M_{1},
\end{aligned}
$$

where the dot denotes differentiation with respect to $t$. Imposing that $\eta(t)=\int{ }^{t} f(\lambda) d \lambda$, we eliminate the spatial dependence of the above equations and obtain

$$
\begin{gathered}
i \dot{M}_{1}=m M_{1}+\eta M_{2}, \\
i \dot{M}_{2}=-m M_{2}+\eta M_{1} .
\end{gathered}
$$

Now we simplify even more the above equations by performing the following identifications:

$$
M_{1} \equiv G_{1} e^{-i m t}, \quad M_{2} \equiv G_{2} e^{i m t},
$$

getting

$$
\dot{G}_{1}=-\eta_{1} G_{2}, \quad \dot{G}_{2}=-\eta_{2} G_{1},
$$


where $\eta_{1} \equiv \eta e^{2 i m t}$ and $\eta_{2} \equiv \eta e^{-2 i m t}$. By deriving the above equations it is easy to conclude that one gets the following second-order equations:

$$
\ddot{G}_{i}-\left(\frac{\dot{\eta}_{i}}{\eta_{i}}\right) \dot{G}_{i}+\eta^{2} G_{i}=0, \quad i=1,2
$$

At this point it is convenient to rescale the $G_{i}$ functions as $G_{i}=\sqrt{\eta_{i}} g_{i}$. One gets finally that

$$
\ddot{g}_{i}+\left[\frac{1}{2}\left(\frac{\ddot{\eta}_{i}}{\eta_{i}}\right)-\left(\frac{\dot{\eta}_{i}}{\eta_{i}}\right)^{2}+\eta^{2}\right] g_{i}=0 .
$$

Note that, in general, these last equations are not somewhat straightforward to solve as asserted in Ref. [12]. However, we note that for a time exponentially decaying force, these equations take the form a Schrödinger equation for the Morse potential in the time variable, without the usual boundary conditions of such kind of equations.

From now on, we will present an extension of the class of time-dependent relativistic systems with exact solutions. For this purpose we begin with the complete Hamiltonian (1) and make the more general ansatz

$$
\psi=\left(\begin{array}{ll}
M_{1}(t) & e^{i F_{1}(q, t)} \\
M_{2}(t) & e^{i F_{2}(q, t)}
\end{array}\right) .
$$

Thus we obtain

$$
\begin{gathered}
i \dot{M}_{1}=\left[\dot{F}_{1}+V_{V}+V_{S}+m\right] M_{1}+\left(F_{2}^{\prime}-V_{P}\right) e^{i\left(F_{2}-F_{1}\right)} M_{2}, \\
i \dot{M}_{2}=\left[\dot{F}_{2}+V_{V}-V_{S}-m\right] M_{2}+\left(F_{1}^{\prime}+V_{P}\right) e^{-i\left(F_{2}-F_{1}\right)} M_{1},
\end{gathered}
$$

where the prime denotes differentiation with respect to $q$. As by construction $M_{i}$ do not depend on $q$, one sees that it is mandatory to get rid of such a dependence in the above equations. As a consequence two classes of solution emerge, corresponding to $F_{1}=F_{2}$ and $F_{1}=-F_{2}$.

Let us analyze the class where $F_{1}=F_{2}=F$. In this case the exponential factor appearing in the equations disappears and, consequently, the only way of getting the off-diagonal terms independent of $q$, is by imposing that

$$
V_{P}=V_{P}(t), \quad F=\theta_{1}(t) q .
$$

On the other hand, the effect of this condition over the diagonal terms results in

$$
V_{S}=V_{S}(t), \quad V_{V}=-\dot{\theta}_{1}(t) q+\theta_{2}(t) .
$$

Note that this class allows the treatment of systems that are at most linear in the spatial coordinate, and includes the system proposed by Landim and Guedes as a particular case. The general equations to be solved in this class are now given by

$$
\begin{aligned}
& i \dot{M}_{1}=\left(\chi_{+}+m\right) M_{1}+\eta_{-} M_{2}, \\
& i \dot{M}_{2}=\left(\chi_{-}-m\right) M_{2}+\eta_{+} M_{1},
\end{aligned}
$$

with $\chi_{ \pm} \equiv \theta_{2} \pm V_{S}$ and $\eta_{ \pm} \equiv \theta_{1} \pm V_{P}$. The above equations can now be decoupled, giving

$$
\begin{aligned}
i \ddot{G}_{1}- & {\left[\chi_{+}+\chi_{-}+i\left(\frac{\dot{\eta}_{1}}{\eta_{1}}\right)\right] \dot{G}_{1}-\left[\dot{\chi}_{+}-\left(\frac{\dot{\eta}_{1}}{\eta_{1}}\right) \chi_{+}-i \eta_{+} \eta_{-}\right.} \\
& \left.+i \chi_{+} \chi_{-}\right] G_{1}=0 \\
i \ddot{G}_{2}- & {\left[\chi_{+}+\chi_{-}+i\left(\frac{\dot{\eta}_{2}}{\eta_{2}}\right)\right] \dot{G}_{2}-\left[\dot{\chi}_{-}-\left(\frac{\dot{\eta}_{2}}{\eta_{2}}\right) \chi_{-}-i \eta_{+} \eta_{-}\right.} \\
& \left.+i \chi_{+} \chi_{-}\right] G_{2}=0
\end{aligned}
$$

where we use the same definition as above for the $G_{i}$ functions and now $\eta_{1} \equiv \eta_{-} e^{2 i m t}$ and $\eta_{2} \equiv \eta_{+} e^{-2 i m t}$. It is easy to verify that the above equations recall that appearing in Eq. (8) in their particular case. Once again it is, in general, not solvable, showing that we have got a formal solution, so that if one wants to solve the problem until the end, one must look for particular cases where these equations have explicit solutions.

From now on we analyze the class where $F_{1}=-F_{2}=F$. In this new class, the exponential factor appearing in the equations holds and, consequently, the only way of getting the off-diagonal terms independent of $q$ in Eqs. (11), is by imposing that

$$
F^{\prime}=-V_{P}(q, t)
$$

Now, the condition of spatial independence of the diagonal terms implies that

$$
V_{V}=V_{V}(t), \quad V_{S}=V_{S}(q, t)=\gamma(t)-\dot{F}(q, t),
$$

where $\gamma(t)$ is an arbitrary function of time. In this case the equation decouples, giving simply

$$
i \dot{M}_{1}=\left(V_{V}+\gamma+m\right) M_{1}, \quad i \dot{M}_{2}=\left(V_{V}-\gamma-m\right) M_{2} .
$$

Now it is easy to obtain the solution to these equations. These are given by

$$
\begin{aligned}
& M_{1}(t)=M_{1}(0) \exp \left(-i\left\{m t+\int^{t}\left[V_{V}(\lambda)+\gamma(\lambda)\right] d \lambda\right\}\right), \\
& M_{2}(t)=M_{2}(0) \exp \left(i\left\{m t-\int^{t}\left[V_{V}(\lambda)-\gamma(\lambda)\right] d \lambda\right\}\right) .
\end{aligned}
$$

At this point some comments are in order. From the above findings, we easily conclude that for this second class of solutions, one really obtains arbitrary nonformal solutions, provided that the remaining integrals can be done. Here it is important to remark that this class of systems allows arbitrary dependence on the spatial and time variables, in contrast to the previous class of systems. 
In what follows, we try to construct an invariant operator of the Lewis and Riesenfeld type [13], which has the above classes of solutions as eigenfunctions. It could be used, as done in Ref. [12], in order to suggest the form of the spinor presented as an ansatz in this work. The invariant obviously must satisfy the equation

$$
\frac{d I}{d t}=\frac{\partial I}{\partial t}-i[I, H]=0
$$

and can be written as

$$
I=\mathcal{A}(t) p+\mathcal{F}(q, t),
$$

where $\mathcal{A}$ and $\mathcal{F}$ are $2 \times 2$ matrices, whose elements, in order to guarantee the validity of Eq. (20), must obey the following set of coupled differential equations:

$$
\begin{aligned}
\mathcal{A}_{11} & =\mathcal{A}_{22}=\mathrm{const}, \quad \mathcal{A}_{12}=\mathcal{A}_{21}=\text { const }, \\
\Lambda_{-}^{\prime} & =-\mathcal{A}_{11} V_{P}^{\prime}, \quad \Sigma_{-}=\mathcal{A}_{12}\left(m+V_{S}\right), \\
\Sigma_{+} & =\Lambda_{+}+\Lambda_{-}-\mathcal{A}_{11} V_{V}^{\prime}, \\
-i \dot{\Sigma}_{-} & =2 \Lambda_{+}-i \mathcal{A}_{12} V_{P}^{\prime}-i \mathcal{A}_{11} V_{S}^{\prime}, \\
i \dot{\Lambda}_{+} & =2 \Sigma_{-} V_{P}+i \Sigma_{-}^{\prime}+i \mathcal{A}_{12} V_{V}^{\prime}+2 \Lambda_{-}\left(m+V_{S}\right), \\
-i \Lambda_{-} & =i \Sigma_{+}^{\prime}+i \mathcal{A}_{11} V_{P}^{\prime}+i \mathcal{A}_{12} V_{S}^{\prime}-2 \Lambda_{+}\left(m+V_{S}\right),
\end{aligned}
$$

where we defined that $\Lambda_{ \pm} \equiv\left(\mathcal{F}_{12} \pm \mathcal{F}_{21}\right) / 2$ and $\Sigma_{ \pm} \equiv\left(\mathcal{F}_{11}\right.$ $\left.\pm \mathcal{F}_{22}\right) / 2$. On the other hand, we are looking for invariant operators, which have the Dirac spinors as eigenfunctions, which warrants further conditions over the elements of the matrices $\mathcal{A}$ and $\mathcal{F}$. This led us to the following conditions, if we consider the eigenvalue equation for the invariant operator acting over the spinor (10) introduced in this work:

$$
\begin{array}{r}
\mathcal{A}_{11}\left(F_{1}^{\prime}+F_{2}^{\prime}\right)+\mathcal{F}_{11}+\mathcal{F}_{22}=\chi_{1}(t), \\
\left(\mathcal{A}_{12} F_{1}^{\prime}+\mathcal{F}_{21}\right)\left(\mathcal{A}_{12} F_{2}^{\prime}+\mathcal{F}_{12}\right)=\chi_{2}(t)
\end{array}
$$

with the functions $\chi_{1,2}$ being arbitrary functions of time. It is not difficult to show that, for the first class, one can obtain a solution, which includes that proposed in Ref. [12] as a particular case, given by

$$
\begin{gathered}
\mathcal{A}_{12}=\mathcal{A}_{21}=\mathcal{F}_{12}=\mathcal{F}_{21}=0, \quad \mathcal{A}_{11}=\mathcal{A}_{22} \equiv \mathcal{A}, \quad \mathcal{F}_{11}=\mathcal{F}_{22} \equiv \mathcal{F}, \\
V_{S}=V_{S}(t), \quad V_{P}=V_{P}(t), \quad V_{V}=\frac{\dot{\mathcal{F}}}{\mathcal{A}} q+\chi(t) .
\end{gathered}
$$

For the second class of wave functions, however, as can be easily realized from the above set of coupled nonlinear equations (22) and the constraints coming from Eq. (23), it is quite difficult to extract simple solutions. For this reason we were not able to verify if there exists a relativistic invariant that is linear in the momentum, which is responsible for the generalized solutions here presented. In fact, since it is typical of nonlinear equations, different solutions of it could lead to independent sets of wave functions. Finally, it is interesting to make some comments: the first one is the observation that the extension of the solutions discussed in this work, by introducing a time-dependent mass, is quite simple, as can be seen from its appearance combined with the time-dependent potentials $V_{S}$ and $V_{V}$. On the other hand, the case of the so-called Dirac oscillator [14] is not directly solvable from the second class of solutions. This happens because when $V_{P}$ is linear in the coordinate, it warrants the need of a $V_{S}$ quadratic in $q$, in order to guarantee the exact solution of the form guessed in this work.

The authors are grateful to FAPESP and CNPq for partial financial support.
[1] R.K. Colegrave and M.S. Abdalla, Opt. Acta 30, 861 (1983); 28, 495 (1981).

[2] H.R. Lewis and K.R. Symon, Phys. Fluids 27, 192 (1984).

[3] C. Farina and A. de Souza Dutra, Phys. Lett. A 123, 297 (1987).

[4] A. de Souza Dutra and B.K. Cheng, Phys. Rev. A 39, 5897 (1989).

[5] B.R. Holstein, Am. J. Phys. 57, 714 (1989).

[6] C.F. Lo, Phys. Rev. A 45, 5262 (1992).

[7] J.Y. Ji, J.K. Kim, and S.P. Kim, Phys. Rev. A 51, 4268 (1995).
[8] I.A. Pedrosa, G.P. Serra, and I. Guedes, Phys. Rev. A 56, 4300 (1997).

[9] I. Guedes, Phys. Rev. A 63, 034102 (2001).

[10] M. Feng, Phys. Rev. A 64, 034101 (2001).

[11] I. Sturzu, Phys. Rev. A 64, 054101 (2001).

[12] R.R. Landim and I. Guedes, Phys. Rev. A 61, 054101 (2000).

[13] H.R. Lewis and W.B. Riesenfeld, J. Math. Phys. 10, 1548 (1969).

[14] M. Moshinsky and A. Szczepaniak, J. Phys. A 22, L817 (1989). 